Article

\title{
A Biomechanical Study for Developing Wearable-Sensor System to Prevent Hip Fractures among Seniors
}

\author{
Gongbing Shan ${ }^{1,2, *}$, Xiang Zhang ${ }^{1,2}$, Mingliang Meng ${ }^{2}$ and Brandie Wilde ${ }^{1}$ \\ 1 Biomechanics Lab, Faculty of Arts \& Science, University of Lethbridge, Lethbridge, AB T1K 3M4, Canada; \\ xiangzhang@xztc.edu.cn (X.Z.); brandie.lea@uleth.ca (B.W.) \\ 2 Department of Physical Education, Xinzhou Teachers' University, Xinzhou 034000, China; \\ mengml@xztc.edu.cn \\ * Correspondence: g.shan@uleth.ca; Tel.: +1-403-329-2683
}

Received: 27 April 2017; Accepted: 27 July 2017; Published: 30 July 2017

\begin{abstract}
As the population ages, falls are becoming a major health problem, not only for those with some degree of balance or mobility impairment, but also among healthy active seniors. Previous studies suggest that the degradation of human sensorimotor function related to age contributes to falls. Hip bones are among the most frequently fractured body parts resulting from falls. Hip fractures are a frequent cause of early death, functional dependence, and high medical care costs. The current prevention method is to use hip protectors. Unfortunately, it often fails to do so because the pocket containing the pad can move away from the area during falls. Additionally, some seniors refuse to use hip protectors because they find them constraining. Hence, a new protector that is only activated during a fall is much desired. The current study explored the possibility via biomechanical analyses for building a wearable sensor system that triggers a mini-airbag system during a fall, i.e., the air-pad is only present for protection when a fall occurs. The results have revealed that two sensors placed on the left and right shoulder would be best for a detection of any-direction fall and could be applied for building a wearable sensor system for prevention of hip fractures resulting from falls.
\end{abstract}

Keywords: aging; sensorimotor degradation; falls; fall-triggered air-pad; threshold

\section{Introduction}

The frequency of falls and fall-related injuries increases with age. As the population ages, falls become one of the major health problems, not only for those with some degree of balance or mobility impairment, but also among healthy active seniors [1-3]. Previous studies in the USA have revealed that falls were the leading cause of external injury among the elderly, accounting for 24 percent of emergency visits [4] and responsible for 70 percent of accidental deaths in persons aged 75 years and older [1]. The estimated expenditure for treatments of fall-related injuries amounts to 20.2 billion US dollars annually [5]. Further, previous studies also revealed that one-third of people 65 years and older experience at least one fall each year [3,6,7]. This severe problem has a significant impact on personal health and medical care costs in older adults. As half of the baby-boom generation crosses into its 60s, falls, and associated costs, are becoming a major problem worldwide.

Hip bones are among the most frequently fractured body parts resulting from falls. They are a frequent cause of early death, functional dependence, and high medical care costs [8]. At the moment, wearable sensor systems tend to collect information such as velocity, acceleration, vibration, and tilt signals to discriminate falls $[9,10]$, however, they are fall detectors, and cannot prevent injuries resulting from falls. Obviously, these fall-detectors can significantly shorten the time for an emergency treatment, but hardly protect seniors during falls. 
The current prevention method is to use hip protectors (Figure 1). The pads are held in position by being incorporated into a garment that is supposed to keep the pad in the right position with respect to the hip area. Unfortunately, it often fails to do so because the pocket containing the pad can move away from the area during falls [11]. Additionally, some seniors refuse to use hip protectors because they find them constraining their daily activities [12]. Hence, a new type of protector that is only activated during a fall is desired. Such an idea can be realized by using a wearable-senor system to provide a fall-triggered air-pad unit (like an air-bag in cars). The unit could be placed within a vest connected to shorts (the air-bag). The core of such a unit is a pyrotechnic gas generator. Such a generator for cars made by SEVA Technologies is only $\sim 100 \mathrm{~mm}$ long with a diameter of $\sim 30 \mathrm{~mm}$, weighs $\sim 300 \mathrm{~g}$ and is less than $€ 10$ [13]. It should be noted that the desired air-pad requires about $1 / 20$ the amount of air that a car airbag does. Technically, such a mini air-pad unit would not change the appearance or weight of a vest significantly.

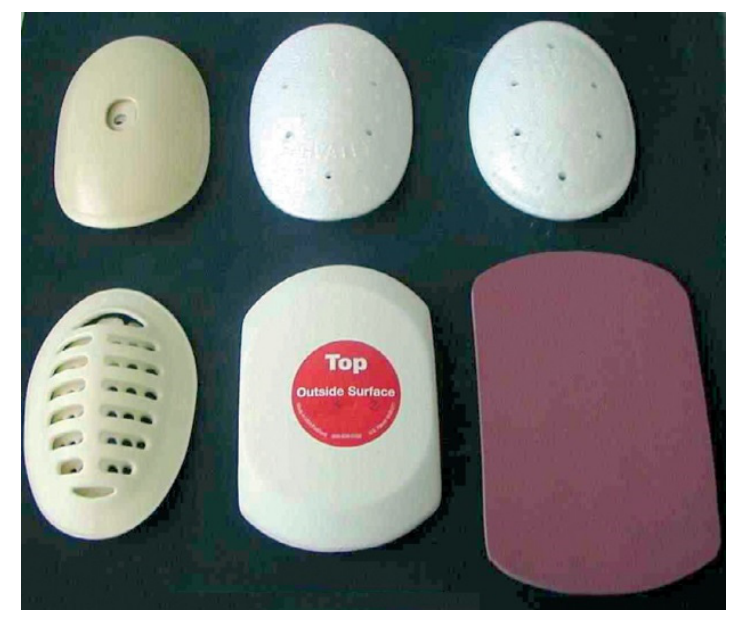

Figure 1. The currently common-used hip protectors for seniors.

There are several existing studies exploring the possibility of wearable systems for injury protection during falls. Most of the previous studies started prototyping without characterization of or comparison between senior daily activities and their possible fall movements [14,15]. Their developments were related to the hardware development, i.e., the focuses were not to explore the reliability of fall detection methods. One potential risk of such an approach would be that the airbag may be activated occasionally during daily activities. There was one study that kinematically compared daily activities and falls [16], but involved only two realistic activities, i.e., walking and sitting down, (the other tested conditions-lying down on a bed and getting out of the bed-would not be practical, i.e., sleeping with airbag). There are too many unknowns which have not been investigated. Biomechanically, for developing such a wearable-sensor system to prevent hip fractures, studies are first required to distinguish between routine daily activities and potential fall situations.

Consequently, for developing a reliable wearable-sensor fall-trigger, fundamental research is needed (1) to quantify the characteristics of seniors' daily activities for establishing movement threshold values for type-of-activity decisions, i.e., normal activity or falling; and (2) to identify sensible places on the body for rapid identification of a fall. Hence, the aim of the current study was to lay the foundation for such a development. It focused on determining the number and location of sensors required on the trunk for characterizing the process of senior falls. 


\section{Materials and Methods}

\subsection{Subjects}

A total of 21 participants (male: $n=12$; female: $n=9$, age 20-35) participated in the study. The study protocol (NSERC Project \#569) was approved by the Human Subjects Research Committee of the University of Lethbridge in accordance with the Tri-Council Policy Statement: Ethical Conduct for Research Involving Humans and all participants completed informed consent documents prior to data collection.

\subsection{Type of Activity}

Senior falls are mainly caused by weak muscles, slow reaction and movement disturbances $[3,8,17]$. For mimicking seniors' motor control conditions, the young subjects with eyes closed were tested at relaxed standing (simulating weak muscles and slow reaction) through a push-fall method (movement disturbances). The relax-falls were protected by foam mats. A test subject was first twisted (rotated) and randomly selected the push direction (forward, backward, downward/sit, left or right). The push-direction and closed eyes simulated the sudden loss of balance. Additionally, an eyes-closed, slow walk was applied for a trip-fall test. The six fall tests (Figure 2) would represent common fall situations among seniors.

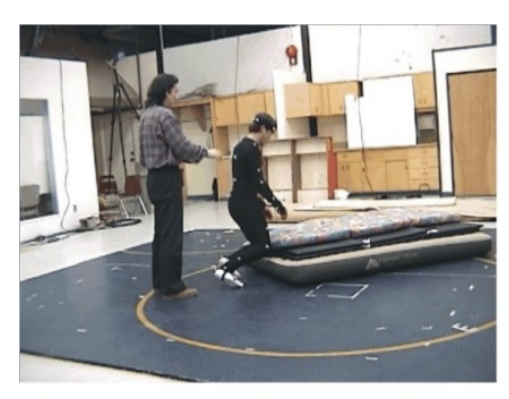

(a)

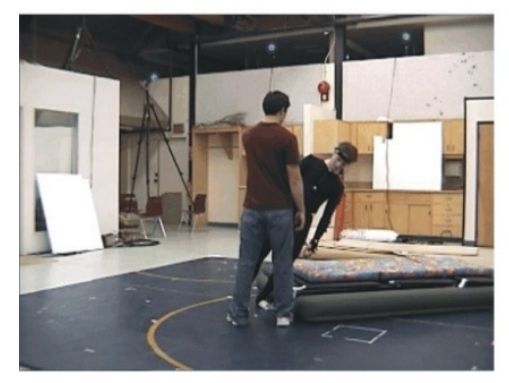

(d)

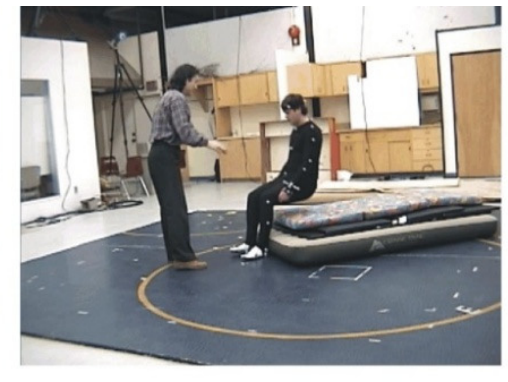

(b)

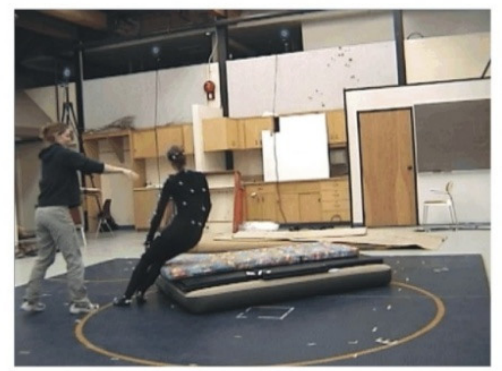

(e)

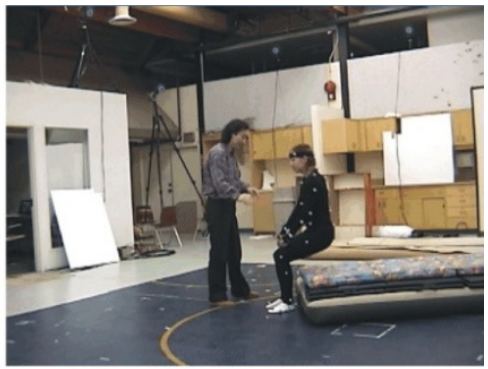

(c)

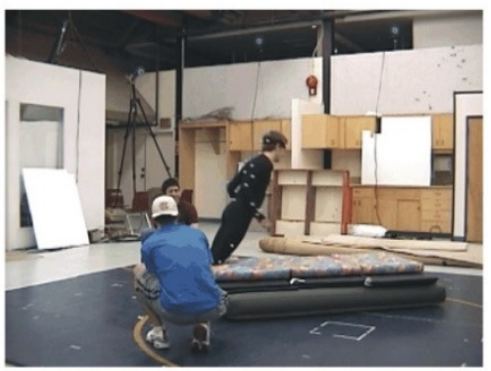

(f)

Figure 2. Motion analysis/quantification of the common type of falls among seniors: (a) a forward fall; (b) a backward fall; (c) a sit fall (downward); (d) a left fall; (e) a right fall; (f) a trip fall.

Seniors' falls often happened during a 'switch' between physical activities, such as slip/trip, sit down/stand up, up-/downstairs or reaching for something [1,18]. Hence, these daily activities, i.e., sitting, standing from a chair, walk with eyes-closed (mimic seniors' weak muscles for their motor control), going upstairs, walking downstairs, and reaching movement, were simulated, captured, and analyzed (Figure 3). Through comparisons between fall and non-fall situations, the number and locations of sensors for a reliable detection could be determined. 


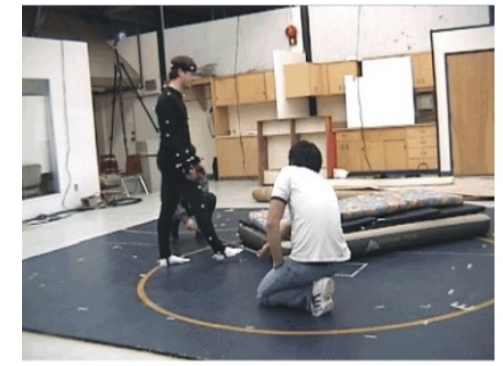

(a)

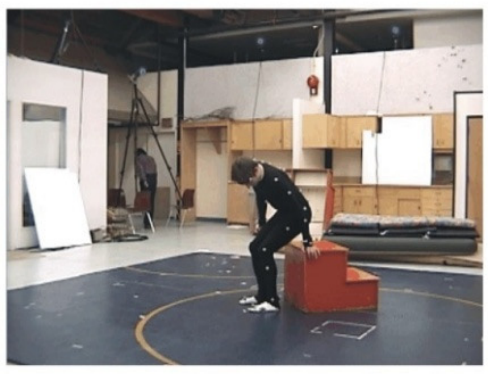

(d)

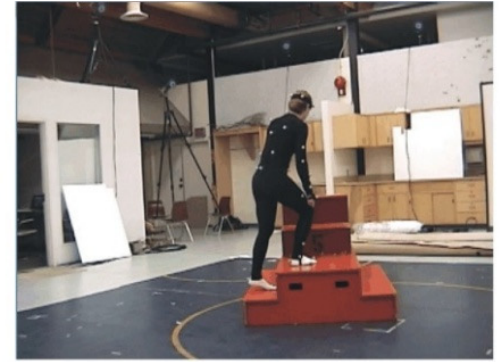

(b)

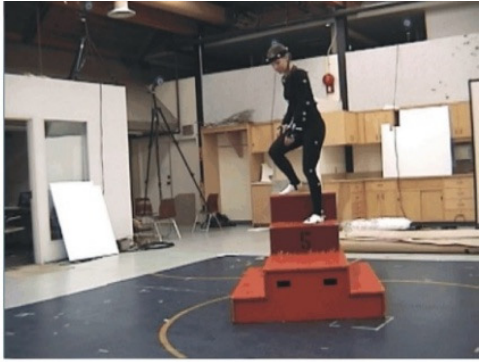

(c)

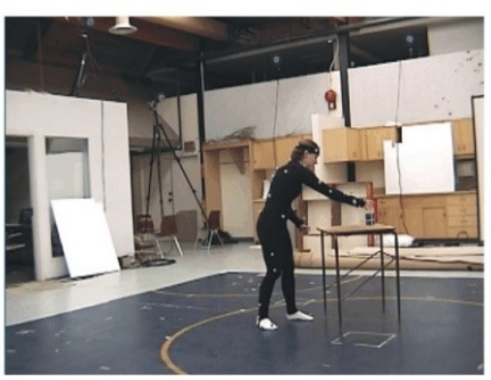

(e)

Figure 3. Motion analysis/quantification of the common daily activities among seniors: (a) a walk with eyes-closed (followed by a trip); (b) going upstairs; (c) walking downstairs; (d) sitting down and standing up; (e) reaching for an object.

\subsection{Three-Dimesional Motion Capture and Biomechanical Modeling}

A 12-camera VICON MX40 motion capture system (Oxford Metrics Ltd., Oxford, UK) was set up to capture the movements. Cameras were placed in a circle around the subjects, creating a capture volume of approximately $5 \times 5 \times 3 \mathrm{~m}$. The motion capture tracked and triangulated the positions of markers made from 3M Super-reflective Tape that are placed on the subject, i.e., the captured data supplied the spatial position of the markers. The use of motion-capture permits considerable freedom of movement without influencing the accuracy of data, so no restrictions were placed on the movement of the subjects within the capture volume and the subjects could perform the selected movement without having to alter their normal body language. Using the manufacturer's specified guidelines, calibration resolution yielded results accurate to $<1 \mathrm{~mm}$. Data was gathered at 250 frames/second, which allows the finest details of movement to be accurately examined.

Forty-four spherical, reflective markers (diameter $=9 \mathrm{~mm}$ ) were placed on each participant (Figures 2 and 3). Forty-two were located at the standard anatomic landmarks necessary to create a 15 segment, full-body biomechanical model [19-21]. The placement of body markers was as follows: four markers on the head (one each on the left and right temples and two on the posterior portion of the parietal bone); six markers on the upper trunk (one each on the sternal notch, xiphoid process, C7 and T10 vertebrae and the left \& right back); seven markers on each upper limb (acromion processes, lateral epicondyles of the humerus, styloid processes of the ulna and radius, third metacarpophalangeal joints, and upper and lower arms); four markers on the pelvis (one each on left and right anterior and posterior superior iliac crests); and six markers on each lower limb (upper thigh, lateral condyle of the tibia, lateral side of the tibia, lateral malleolus of the fibula, calcaneal tuberosity, head of halluces) (Figure 4). The additional two markers were placed at the left and right bottom of the back (representing the left and right bottom of a vest) for a possible consideration of sensor positions. 


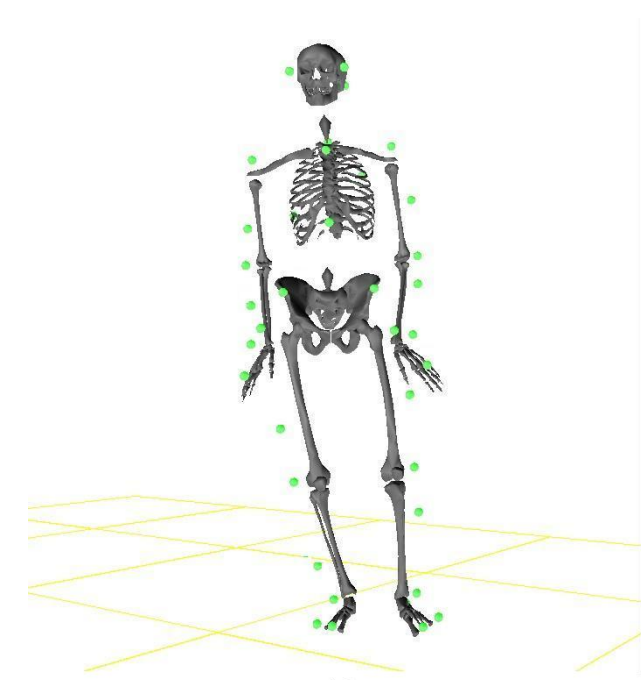

(a)

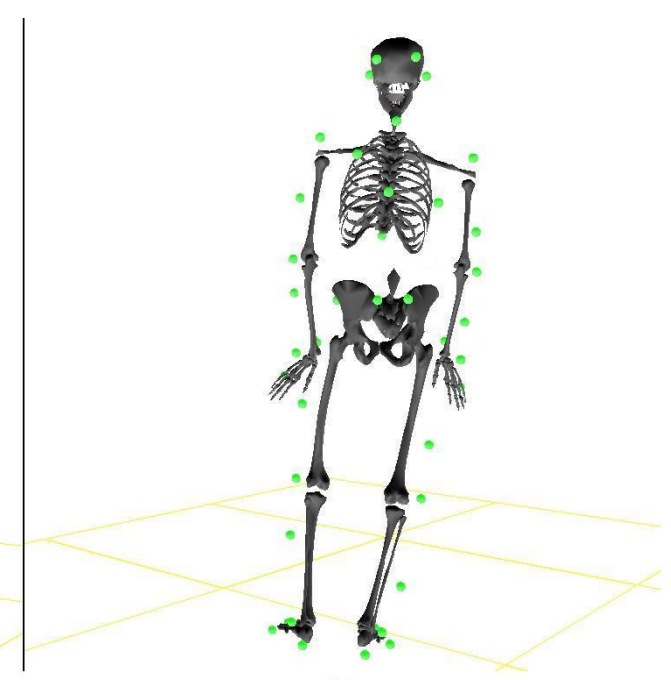

(b)

Figure 4. Placement of the 42 reflective markers for full-body biomechanical modeling [19-21]: (a) a front view; (b) a back view. The asymmetric placement of the markers on limbs was used for distinguishing the left and right side of the body during motion capture.

After positional data was captured, it was applied to a 15-segment biomechanical model. The 15 segments were head, upper torso, lower torso, upper arms, forearms, hands, thighs, legs, and feet. Data was filtered using a five-point moving average filter (weighted: 1-3-4-3-1). The model calculations supplied the positions, speeds, and accelerations of each marker, as well as all joint angles: flexion/extension (Flex/Ext), abduction/adduction (Ab/Add), and rotation, i.e., the motor controls of fall and non-fall situations. A 3D sample of side-fall characterized by the 15-segment biomechanical body model was shown in Figure 5. In 3D body modeling, inertial characteristics for each segment were determined by applying anthropometric regression equations with individual body mass, body height, and gender as input parameters [19]. Using the biomechanical model, sensitive sensor locations and threshold values for distinguishing daily activities and falls were quantified.

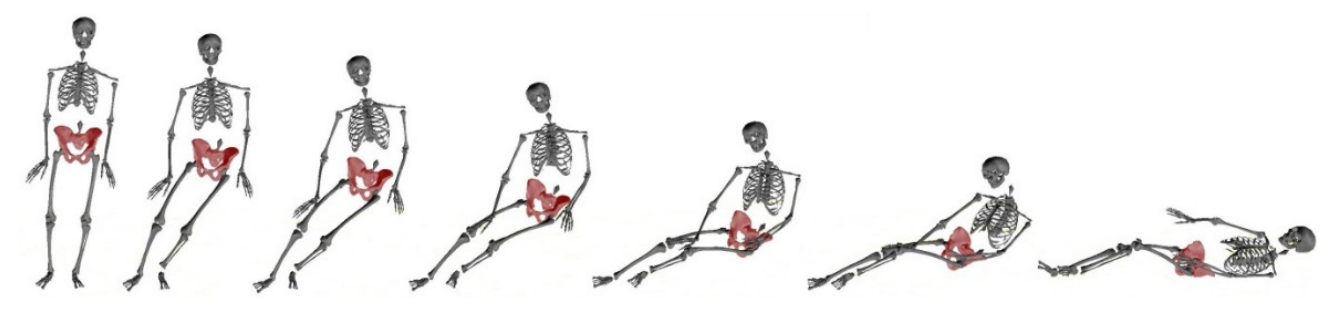

Figure 5. Biomechanical modeling based on 3D motion capture-a typical fall to the left.

\subsection{Markers of Interest and Data Analysis}

Since the wearable-senor system (the fall-trigger) and the main part of the air-pad unit could only be placed within a vest, the markers of interest (possible sensors' positions) were those on the trunk: 7th cervical vertebrae (C7), clavicle, sternum, left shoulder (LSho), right shoulder (RSho), 10th thoracic vertebrae (T10), left scapula/back (LB), right scapula/back (RB), lower left back (LLB), and lower right back (LRB) (Figure 6). Kinematic data (i.e., max speed, max acceleration, and the duration of fall) were quantified by the 15-segment model. The kinematic data obtained were analyzed using SPSS Statistics v.22.0 (SPSS Inc., Chicago, IL, USA). Results were presented using descriptive statistics. Further, for developing a practical device, the focuses would be on convenience (minimal sensors required) and reliability (avoiding the cumulative Type I and II error rate from complicated statistical analyses). Therefore, independent $t$-tests were applied to contrast differences between fall and non-fall 
situations, aiming at identifying one or two potential sensitive locations for the development. Statistical significance was defined as $p<0.05$.

In order to minimize the air-pad system (i.e., minimizing the amount of air needed for a protection), the short should consist of three air-pads, left, right, and rear. Therefore, the other function of the wearable sensor-system should be to identify the fall direction. As such only one of the three pads would be inflated during a fall.

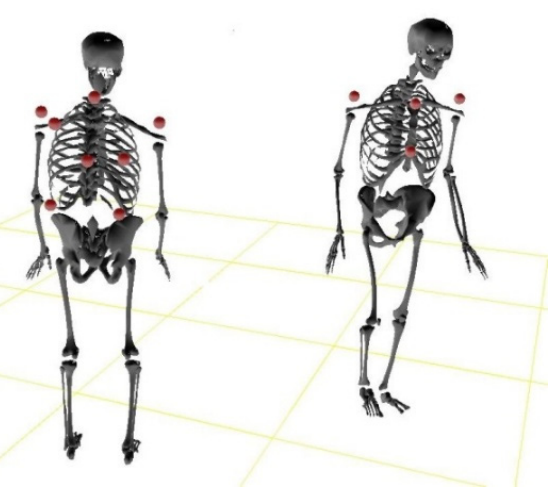

Figure 6. Markers of interests-possible positions under investigation for wearable-sensors: (left): back view of the possible positions; (right): the front view of the possible positions.

\section{Results}

Three-dimensional motion analysis of selected parameters revealed that acceleration was not a reliable parameter for distinguishing senior falls from the common daily activities, especially there was no significant difference for maxima of acceleration between downstairs and fall-down (sit-fall) $(p>0.05)$. However, speed maximum was identified as a reliable parameter. Comparing the daily activities to the fall situations (Figure 7), a highly significant difference $(p<0.000$, the adjusted $p$-value using Bonferroni for multi-comparisons is $p=0.004[p=0.05 / 12=0.004]$ ) was observed in each marker of interest. The speed maxima reached during fall situations were $2-4$ times that of daily activities.

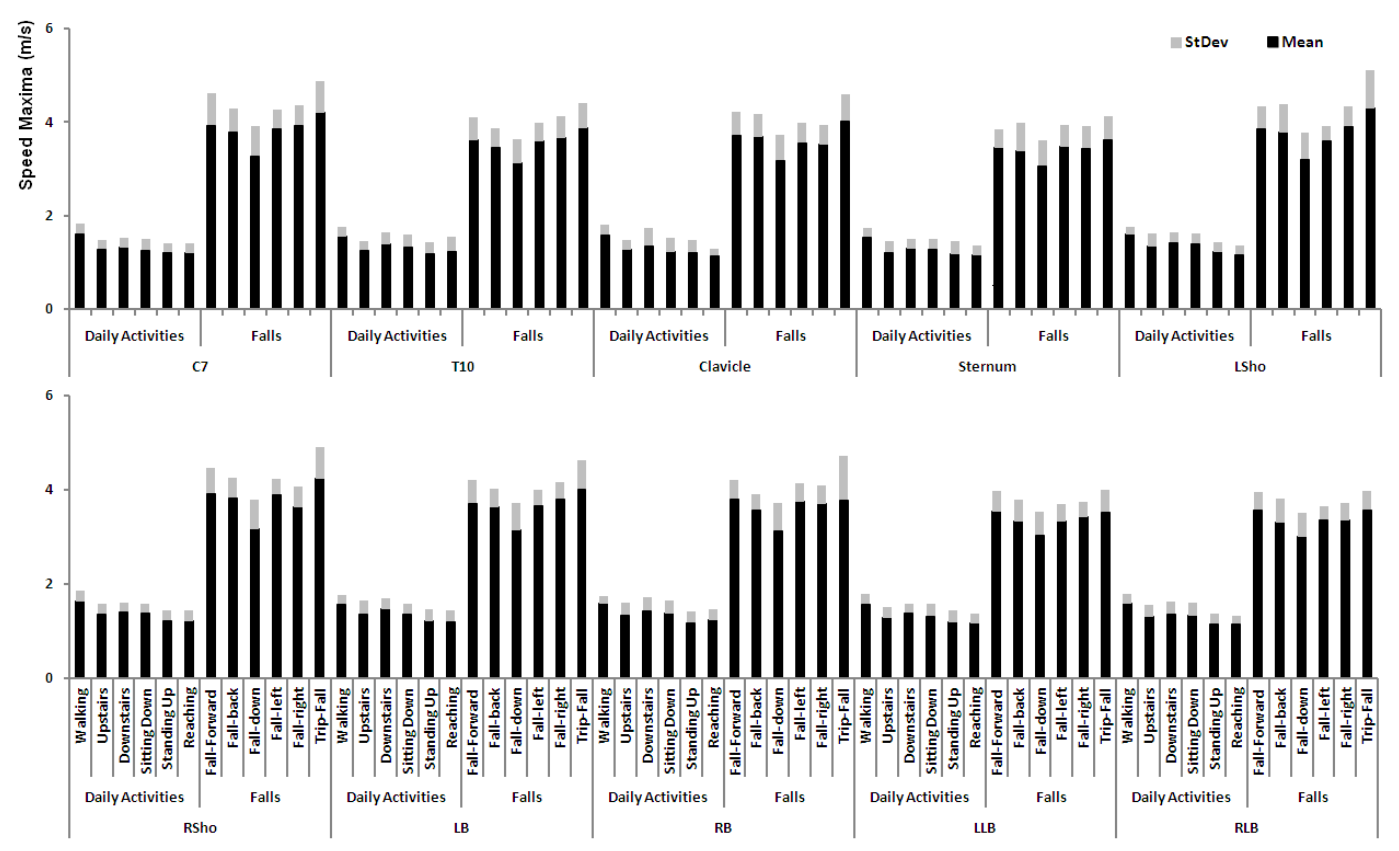

Figure 7. Speed maximum (mean and StDev [standard deviation]) at each marker of interest for daily activities and fall situations. 
Further, the statistic tests indicated that LSho and RSho markers could be used to identify fall directions. For a fall left, the speed maximum of RSho was significantly high than that of LSho $(p<0.05)$, vice versa for a fall right. For the remaining fall situations, there were no significant differences $(p>0.05)$.

In general, falls finished within one second. There were obviously differences among the durations across all fall types (Table 1). The shortest fall was found to be fall down (i.e., sit fall), taking about $0.7 \mathrm{~s}$; while the longest fall, i.e., fall left, needed about $0.9 \mathrm{~s}$ to finish.

Table 1. The duration (mean \pm standard deviation) of fall at each fall situations

\begin{tabular}{ccccccc}
\hline Duration & Fall-Forward & Fall-Back & Fall-Down & Fall-Left & Fall-Right & Trip-Fall \\
\hline Fall Time (s) & $0.92 \pm 0.21$ & $0.79 \pm 0.18$ & $0.72 \pm 0.16$ & $0.94 \pm 0.25$ & $0.93 \pm 0.23$ & $0.76 \pm 0.13$ \\
\hline
\end{tabular}

The speed maxima occurrence in relation to elapsed time can be seen in Table 2. It was concluded that max speed happened almost at the end of a fall ( $~ 90 \%$ of the duration). Further analysis showed that, when the maximum (i.e., $2.08 \mathrm{~m} / \mathrm{s}$ ) of all speeds measured during the common daily activities as a threshold value, the percentage of time elapsed dropped to $\sim 60 \%$ of the duration.

Table 2. Timing characteristics of the markers of interest during falls

\begin{tabular}{ccc}
\hline Marker & Percentage of Time Elapsed at Max Speed & Percentage of Time Elapsed at 2.1 m/s \\
\hline C7 & $90.2 \pm 0.5$ & $58.2 \pm 0.3$ \\
T10 & $88.8 \pm 0.4$ & $60.3 \pm 0.5$ \\
Clavicle & $86.5 \pm 0.6$ & $59.3 \pm 0.3$ \\
Sternum & $89.7 \pm 0.4$ & $60.4 \pm 0.2$ \\
LSho & $89.3 \pm 0.3$ & $57.9 \pm 0.6$ \\
Rsho & $89.6 \pm 0.3$ & $57.7 \pm 0.2$ \\
LB & $89.8 \pm 0.4$ & $58.9 \pm 0.3$ \\
RB & $88.9 \pm 0.3$ & $59.2 \pm 0.4$ \\
LLB & $87.2 \pm 0.2$ & $57.7 \pm 0.3$ \\
LRB & $87.4 \pm 0.4$ & $57.5 \pm 0.5$ \\
\hline
\end{tabular}

In summary, due to insignificant differences of maximal acceleration between some falls (e.g., fall-down/sit-fall) and non-falls (e.g., downstairs), acceleration could not reliably tell if there is a fall or a daily activity. When maximal speed during falls is used, the identification problem is solved; but, there is not enough time left for inflating an airbag. The issue could be solved by applying the maximal speed measured in daily activities as a threshold value. For identifying fall directions, the two reliable markers (locations of sensors) are the left and right shoulders.

\section{Discussion}

The purposes of this study were to: (1) quantitatively determine the number and location of wearable sensors required on trunk for characterizing senior falls using a 15-segment, full-body biomechanical model; (2) examine and compare kinematic differences between daily activities and fall situations; and (3) identify the fall direction in order to minimize the desired air-pad system.

The frequency of falls and fall-related injuries among seniors are becoming a major problem worldwide. The majority of previous researches have tended to focus on prevention related to balance ability of seniors and what causes a fall to occur [7,17,22], but what if the inevitable does take place? It is essential to determine fall characteristics and how they differ from that of normal activities. The current state of such studies is well documented by Schwickert's recent review article, which examined 96 selected studies from numinous electronic databases [23]. Published evidence-based support for commercially available fall detection devices is still lacking. A worldwide study is needed to address fundamental issues such as incident verification and fall quantification. 
In the current study, low reliability was found for using max acceleration reached during a fall to detect a loss of balance. The results somehow match the divergence results among previous studies [23], which suggest that the raw signals from accelerometers may not be applied directly. An algorithm for post-data processing should be developed.

Highly significant differences of max speeds were found between the fall situations and the normal activities. The results of the current study suggest that fall detection could be reliably achievable. Since speed is an overtime effect of acceleration (integration of acceleration over time), acceleration sensors could be used to develop a sensory-trigger device. A vest containing such wearable sensors would detect fall activity and inflate mini-airbag system in the event of a fall. The concern of using max speed is that the maximum occurs too late in the event of a fall ( $\sim 90 \%$ of the duration), minimal time would be allocated for airbag deployment. For overcoming the methodological pitfall, a threshold value (i.e., $2.1 \mathrm{~m} / \mathrm{s}$ ) could be applied. The current study has revealed that the value would indicate a high likelihood that a fall was taking place. The difference in time between the beginning of a fall and the reaching of the threshold is between 0.2 and $0.3 \mathrm{~s}$ (Tables 1 and 2). This time span is long enough for inflating an airbag (i.e., less than $0.1 \mathrm{~s}$ needed) [24]. For more reliability, the threshold value could even be set at $2.5 \mathrm{~m} / \mathrm{s}$ without change of a full inflation.

The number and locations found to reach the highest sensibility of detection were to position two sensors on the left and right shoulder (LSho and RSho). These positions would be recommended for earliest fall direction detection. The threshold value of $2.1 \mathrm{~m} / \mathrm{s}$ however, would suggest that the sensors be placed on LSho and RSho to allow the largest time frame to distinguish between falls (including left and right falls) and daily activities. Time is an issue with a fall duration of approximately one second. Adequate foreknowledge of a fall enhances the ability to deploy the airbag mechanism with substantial time to be an effective intervention. Though a custom anatomical airbag device would need to be fabricated, it can be expected that such a device would not be created to deploy any quicker than that used for automobiles (i.e., less than $0.1 \mathrm{~s}$ ). With this in mind, sensor locations that provide a reliable detection of fall direction would be vital for both fall protection and users' convenience, i.e., minimization required for a user-friendly wearable-senor unit.

For further software development, one could employ a learning model based on an artificial neural network (ANN). ANNs can model complex relationships, such as redundancy and nonlinearity through a machine learning algorithm. The model can be trained using the two sensors' input and fall as well as non-fall activities. The trained ANN can make more reliable predictions. Most importantly, such a model could improve prediction accuracy through continuous updates (re-trainings based on more uses [more data]).

Like most lab-based investigations, there could be limitations on the application of the results. One potential limitation could be the difference between the shoulder markers (tightly touched on shoulders) in lab-test situation and sensors in the vest (small sliding possible). Further studies are needed to confirm: (1) the effects of the possible sliding movement on detecting reliability and (2) if additional parameters are needed to restore the reliability.

\section{Conclusions}

Discrimination between falls and daily activities is possible using a speed threshold value of $2.1 \mathrm{~m} / \mathrm{s}$. Two sensors placed on the left and right shoulder would be best for detection of a fall in any-direction. The current study lays a foundation for building a wearable sensor system for prevention of hip fractures resulting from falls. Not only would this assist the victim, but also reduce the health costs related to resultant injury.

Acknowledgments: The research project was supported by National Sciences and Engineering Research Council of Canada (NSERC), Xinzhou Teachers' University and 2016 Shanxi Scholarship Council of China.

Author Contributions: G.S., X.Z., M.M., and B.W. conceived and designed the experiments; G.S., X.Z., and B.W. performed the experiments; G.S., X.Z., M.M., and B.W. analyzed the data; G.S. and X.Z. contributed reagents/materials/analysis tools; G.S., X.Z., and B.W. wrote the paper. 
Conflicts of Interest: The authors declare no conflict of interest.

\section{References}

1. Greenhouse, A.H. Falls among the elderly. In Clinical Neurology of Aging; Albert, M.L., Ed.; Oxford University Press: London, UK, 1994.

2. Shan, J.; Fu, Y.; Dunn, B.; Shan, G. A Novel Measurement System for Quantitative Assessment of Age-Related Sensorimotor Degradation. Biomed. Eng. Appl. Basis Commun. 2009, 21, 17-28. [CrossRef]

3. Di Rosa, M.; Hausdorff, J.M.; Stara, V.; Rossi, L.; Glynn, L.; Casey, M.; Burkard, S.; Cherubini, A. Concurrent validation of an index to estimate fall risk in community dwelling seniors through a wireless sensor insole system: A pilot study. Gait Posture 2017, 55, 6-11. [CrossRef] [PubMed]

4. Burt, C.W.; Fingerhut, L.A. Injury visits to hospital emergency departments: United States, $1992-95$. Vital Health Stat. 1998, 13, 1-76.

5. Sculpo, T.P. Falling doesn't have to be seniors' last trip, in USA Today. USA TODAY NEW YORK 1998, 127, 32-33.

6. Edelberg, H. Falls and function. How to prevent falls and injuries in patients with impaired mobility. Geriatrics 2001, 56, 41-45. [PubMed]

7. Vieira, E.R.; Palmer, R.C.; Chaves, P.H. Prevention of falls in older people living in the community. BMJ 2016, 353, 1419. [CrossRef] [PubMed]

8. Geusens, P.; Milisen, K.; Dejaeger, E.; Boonen, S. Falls and fractures in postmenopausal women: A review. J. Br. Menopause Soc. 2003, 9, 101-106. [CrossRef] [PubMed]

9. Zhang, T.; Wang, J.; Xu, L.; Liu, P. Fall detection by wearable sensor and one-class SVM algorithm. In Intelligent Computing in Signal Processing and Pattern Recognition; Springer: Berlin/Heidelberg, Germany, 2006; pp. 858-863.

10. Mubashir, M.; Shao, L.; Seed, L. A survey on fall detection: Principles and approaches. Neurocomputing 2013, 100, 144-152. [CrossRef]

11. Hill, K.; Schwarz, J.; Flicker, L.; Carroll, S. Falls among healthy, community-dwelling, older women: A prospective study of frequency, circumstances, consequences and prediction accuracy. Aust. N. Z. J. Public Health 1999, 23, 41-48. [CrossRef] [PubMed]

12. Minns, J.; Dodd, C.; Gardner, R.; Bamford, J.; Nabhani, F. Assessing the safety and effectiveness of hip protectors. Nurs. Stand. 2004, 18, 33-38. [CrossRef] [PubMed]

13. SEVA-Technologies. Next Generation Gas Generators. Available online: http://www.seva-technologies. com/ (accessed on 16 August 2016).

14. Lemus, D.; Vallery, H. Towards Gyroscopic Balance Assistance: Proof of Concept. In Proceedings of the 36th Annual International Conference of the IEEE Engineering in Medicine and Biology Society (EMBC14), Chicago, IL, USA, 26-30 August 2014.

15. Delft University of Technology. Unique 'Fall Airbag' to Protect Elderly From Sustaining Injuries After a Fall. Available online: https:/ /www.tudelft.nl/en/current/latest-news/article/detail/ouderen-beschermdtegen-letsel-bij-vallen-met-unieke-valairbag-1/ (accessed on 6 June 2017).

16. Tamura, T.; Yoshimura, T.; Sekine, M.; Uchida, M.; Tanaka, O. A Wearable Airbag to Prevent Fall Injuries. IEEE Trans. Inf. Technol. Biomed. 2009, 13, 910-914. [CrossRef] [PubMed]

17. Shan, G.; Wilde, B. The selection of pretest states and parameters in identifying the age effect through the center of pressure measurement. Res. Sports Med. 2003, 11, 187-201. [CrossRef]

18. Fuller, G.F. Falls in the elderly. Am. Fam. Phys. 2000, 61, 2159-2168.

19. Shan, G.; Bohn, C. Anthropometrical data and coefficients of regression related to gender and race. Appl. Ergon. 2003, 34, 327-337. [CrossRef]

20. Shan, G.; Westerhoff, P. Soccer: Full-body kinematic characteristics of the maximal instep soccer kick by male soccer players and parameters related to kick quality. Sports Biomech. 2005, 4, 59-72. [CrossRef] [PubMed]

21. Shan, G.; Visentin, P.; Zhang, X.; Hao, W.; Yu, D. Bicycle kick in soccer: Is the virtuosity systematically entrainable? Sci. Bull. 2015, 60, 819-821. [CrossRef]

22. Dunn, B.; Bocksnick, J.; Hagen, B.; Fu, Y.; Li, X.; Yuan, J.; Shan, G. Impact of Exercise on Seniors' Motor Control Response to External Dynamics. Res. Sports Med. 2008, 16, 39-55. [CrossRef] [PubMed] 
23. Schwickert, L.; Becker, C.; Lindemann, U.; Marechal, C.; Bourke, A.; Chiari, L.; Helbostad, J.L.; Zijlstra, W.; Aminian, K.; Todd, C.; et al. Fall detection with body-worn sensors. Zeitschrift für Gerontologie und Geriatrie 2013, 46, 706-719. [CrossRef] [PubMed]

24. Ravop, N. Allen K. Breed, 72, a Developer of Air Bag Technology for Cars. Available online: http:/ / www. nytimes.com/2000/01/14/business/allen-k-breed-72-a-developer-of-air-bag-technology-for-cars.html (accessed on 26 March 2016).

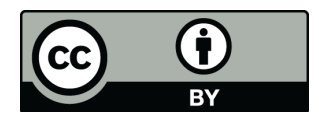

(C) 2017 by the authors. Licensee MDPI, Basel, Switzerland. This article is an open access article distributed under the terms and conditions of the Creative Commons Attribution (CC BY) license (http://creativecommons.org/licenses/by/4.0/). 